\title{
Vergleich der Motivationsprofile von Scrum- Teammitgliedern mit dem Agilen Manifest zur Entwicklung von Gamification-Strategien
}

David Kessing und Manuel Löwer

Agile Methoden sind in der industriellen Anwendung Stand der Technik, wobei Scrum das am häufigsten verwendete Prozess-Framework zur effektiven Entwicklung komplexer Produkte ist. Entworfen wurde Scrum von Ken Schwaber und Jeff Sutherland, die ebenfalls 2001 das agile Manifest mitentwickelten, welches die vier Werte und zwölf Prinzipien für die Arbeit mit agilen Methoden definiert. Scrum zeichnet sich unter anderem durch flache Hierarchien aus. Hierdurch erhalten Mitarbeitende zunehmend Verantwortung und es entsteht aufgrund der vermehrt stattfindenden Kommunikation eine erhöhte Transparenz in allen Prozessen. Diese Eigenschaften bringen viele Vorteile aber auch Herausforderungen mit sich.

Einerseits kann hohe Transparenz durch Offenlegung der Arbeit zu Unsicherheit seitens der Mitarbeitenden führen, andererseits sorgt die erhöhte Verantwortung auch für einen größeren Einfluss der einzelnen Team-Mitglieder auf das Ergebnis der Arbeitsprozesse. Die Motivation und Leistungsbereitschaft der Mitarbeitenden sind demzufolge ausschlaggebend für die erfolgreiche Produktentwicklung mit Scrum.

Gamification ist ein neuer, vielversprechender Ansatz zur Steigerung der Motivation und wird dabei definiert als "die Verwendung von Spiel-Designelementen in Nicht-Spielkontexten".

Die dieser Veröffentlichung zugrundeliegende Forschung bildet die Grundlage zur Entwicklung von dedizierten Gamification-Strategien mit dem Ziel der Optimierung von Motivation und folglich der Leistung der Mitarbeitenden im Scrum-Entwicklungsprozess.

Keywords: Agile Methoden, Scrum, Gamification, Agiles Manifest, Motivation

\section{Einleitung}

Die moderne Produktentwicklung steht vor neuen Herausforderungen. Die zunehmende Geschwindigkeit der gesellschaftlichen Veränderung impliziert schnell wechselnde Anforderungen und damit kürzere Entwicklungszyklen, um Kundenzufriedenheit und Produktrelevanz sicherzustellen (Feldhusen 2009). 
Zur Bewältigung dieser Entwicklung wurde im Jahr 2001 das Konzept der agilen Methoden etabliert, das im sogenannten Agilen Manifest vier Leitwerte und zwölf Prinzipien definiert (Beck et al. 2001).

Die am häufigsten verwendete Agile Methode ist Scrum (CollabNet 2019). Wesentlich für dieses Konzept sind Selbstorganisation, Transparenz und Flexibilität eines jeden Mitarbeiters. So ist die Motivation der Mitarbeiter zur Disziplin und zur Übernahme von Verantwortung in agilen Methoden besonders wichtig (Paetzold 2016).

Gamification ist ein moderner Ansatz zur Steigerung der Motivation durch den Einsatz von "Spieldesign-Elementen in Nicht-Spielkontexten" (Deterding 2011). Im Hinblick auf die beschriebene Entwicklung der Gesellschaft könnte Gamification am Arbeitsplatz in Zukunft eine Schlüsselrolle spielen, da sie moderne Möglichkeiten aufzeigt, motivationalen Herausforderungen zu begegnen (Grünberger 2014).

Diese Forschung leistet drei wesentliche Beiträge: Erstens, eine Gamification-Klassifizierung des Agilen Manifests. Zweitens, eine Umfrage zur Bewertung der gefundenen Hypothese und drittens eine Aufschlüsselung von Scrum-Events mit Fokus auf das Entwicklungsteam mit Ableitung konkreter Gamification-Strategien.

\section{Theoretische Grundlagen}

Scrum ist ein iterativer Produktentwicklungs-Prozess mit sich wiederholenden Zyklen während eines Projekts, um die Möglichkeit zu haben, flexibel auf sich ändernde Anforderungen zu reagieren (Beedle 1999). Mit jedem dieser sich wiederholenden Zyklen wird ein Bestandteil des Endprodukts - ein sogenanntes Inkrement - fertiggestellt. Daher wird das Scrum-Framework auch als iterativ-inkrementeller Entwicklungsprozess bezeichnet (Maximini 2018). Der Kernprozess von Scrum ist der sogenannte Sprint, der 2-4 Wochen dauert und aus der produktiven Arbeit am Inkrement besteht (Krieg 2015). Neben dem Sprint gibt es noch einige Unterprozesse. Das Sprint Planning ist der initiale Start eines Scrum-Zyklus und definiert das Inkrement mit den Aufgaben, die im kommenden Sprint bearbeitet werden sollen. Während des Sprints wird jeden Tag ein Daily Scrum von max. 15 Minuten vom Entwicklungsteam durchgeführt, um über den Fortschritt der Aufgaben und eventuelle Probleme zu sprechen. Nach einer SprintPhase findet das Sprint-Review statt, bei dem das Inkrement den verantwortlichen Stakeholdern präsentiert wird. Zusätzlich findet nach dem Sprint eine Sprint Retrospektive statt, in der neben den Projektinhalten auch methodische und prozessuale Verbesserungen besprochen werden, wie z. B. zwischenmenschliche Konflikte (Dräther 2019). 
Der Begriff Gamification tauchte erstmals 2002 in einem Paper des Unternehmensberaters Nick Pelling auf und hat im Laufe der Zeit in vielen Bereichen immer mehr Aufmerksamkeit erlangt. Im Jahr 2011 fand eine erste wissenschaftliche Konferenz zum Thema statt (Fleisch ${ }^{\circ} 2018$ ). Gamification ist ein relativ junger Ansatz, der im Bereich der Softwareentwicklung seinen Anfang nahm. Mittlerweile werden Gamification-Methoden bereits in vielen Unternehmen erfolgreich eingesetzt (Ellenberger 2020, Reiners 2015).

Das Konzept der Gamification versucht, dieses inhärente Potenzial von Spielen sinnvoll und gezielt zu nutzen. Ziel ist es, die Motivation von Mitarbeitern oder Nutzern zu steigern, indem neue Anreize geboten werden, die das Interesse an Aktivitäten erhöhen und die Bewältigung von Herausforderungen attraktiver machen sollen. Durch die systematische Gestaltung von Gamification-Strategien werden positive Motivationspotenziale erschlossen. Anderie konkludiert überdies weitere Vorteile im Einsatz von Gamification in der Wertschöpfung:

"Gamification beschleunigt Entwicklungsprozesse der Digitalisierung für kulturelle und wirtschaftliche Güter und maximiert den zu verwaltenden Mehrwert." (Anderie 2017)

Verschiedene Frameworks versuchen, Gamification-Techniken durch die angesprochenen Motivationstreiber und unterschiedliche "Spielertypen" zu strukturieren. Relevante aktuelle Ansätze sind Gamified UK von Marczewski, Gamification by Design von Zichermann et al., How to gamify von Morschheuser et al. und Actionable Gamification von Chou (Marczewski 2015, Zichermann 2011, Morschheuser 2017, Chou 2016).

Chou beobachtet in Actionable Gamification acht motivierende Gamification Core Drives von Videospielen, die zur Motivation der Nutzer beitragen:

- Epic Meaning and Calling (Gefühl eines höheren Sinns)

— Entwicklung und Errungenschaft (Gefühl des ständigen Fortschritts)

— Befähigung zur Kreativität und Feedback (Drang zur Selbstverwirklichung)

- Besitz und Eigentum (Wunsch, Eigentum zu bilden)

— Sozialer Einfluss und Verbundenheit (Gefühl der sozialen Akzeptanz)

- Knappheit und Ungeduld (Wunsch, etwas zu erreichen, was derzeit nicht verfügbar ist)

- Unvorhersehbarkeit und Neugier (Drang, unvorhersehbare Situationen zu vermeiden) 


\section{- Verlust und Vermeidung (Drang, Verluste zu vermeiden)}

Ein Kontext, der mit diesem Framework hinsichtlich der Erfüllung der Core Drives analysiert wird, kann in einem Achteck-Graphen (Octalysis) visualisiert werden, wie in Abbildung 1 dargestellt. Da das Framework von Chou ein intuitives Visualisierungswerkzeug und eine hohe Anzahl an erfassten Gamification-Techniken bietet, wird dieser Ansatz in der folgenden Untersuchung verwendet.

\section{Analyse des Agilen Manifests}

Im Folgenden werden die vier Werte und zwölf Prinzipien des Agilen Manifests auf die Gamification Core Drives von Chou abgebildet. Jedem agilen Wert werden zwei Gamification Core Drives basierend auf seinem Inhalt zugeordnet, während jedem agilen Prinzip nur ein Gamification Core Drive zugeordnet wird. (vgl. Tabelle 1 und 2).

Tabelle 1: Zuordnung der Prinzipien des Agilen Manifests zu den acht Octalysis Core Drives

\begin{tabular}{|c|c|}
\hline Prinzipien des Agilen Manifests: & $\begin{array}{l}\text { Zugeordnetes } \\
\text { Core Drive: }\end{array}$ \\
\hline $\begin{array}{l}\text { Unsere höchste Priorität ist es, den Kunden durch frühe und kontinuierliche Auslie- } \\
\text { ferung wertvoller Software zufrieden zu stellen. }\end{array}$ & Epic Meaning \\
\hline $\begin{array}{l}\text { Heisse Anforderungsänderungen selbst spät in der Entwicklung willkommen. } \\
\text { Agile Prozesse nutzen Veränderungen zum Wettbewerbsvorteil des Kunden. }\end{array}$ & Unpredictability \\
\hline $\begin{array}{l}\text { Liefere funktionierende Software regelmäßig innerhalb weniger Wochen } \\
\text { oder Monate und bevorzuge dabei die kürzere Zeitspanne. }\end{array}$ & Accomplishment \\
\hline $\begin{array}{l}\text { Fachexperten und Entwickler müssen während des Projektes täglich zusammenar- } \\
\text { beiten. }\end{array}$ & Social Influence \\
\hline $\begin{array}{l}\text { Errichte Projekte rund um motivierte Individuen. Gib ihnen das Umfeld und die Un- } \\
\text { terstützung, die sie benötigen und vertraue darauf, dass sie die Aufgabe erledigen. }\end{array}$ & Empowerment \\
\hline $\begin{array}{l}\text { Die effizienteste und effektivste Methode, Informationen an und innerhalb eines } \\
\text { Entwicklungsteams zu übermitteln, ist im Gespräch von Angesicht zu Angesicht. }\end{array}$ & Social Influence \\
\hline Funktionierende Software ist das wichtigste Fortschrittsmaß. & Accomplishment \\
\hline $\begin{array}{l}\text { Agile Prozesse fördern nachhaltige Entwicklung. Die Auftraggeber, Entwickler und } \\
\text { Benutzer sollten ein gleichmäßiges Tempo auf unbegrenzte Zeit halten können. }\end{array}$ & Epic Meaning \\
\hline Ständiges Augenmerk auf technische Exzellenz und gutes Design fördert Agilität. & Epic Meaning \\
\hline Einfachheit --die Kunst, die Menge nicht getaner Arbeit zu maximieren -- ist essenziell. & Avoidance of Loss \\
\hline $\begin{array}{l}\text { Die besten Architekturen, Anforderungen und Entwürfe entstehen durch selbstor- } \\
\text { ganisierte Teams. }\end{array}$ & Empowerment \\
\hline $\begin{array}{l}\text { In regelmäßigen Abständen reflektiert das Team, wie es effektiver werden kann und } \\
\text { passt sein Verhalten entsprechend an. }\end{array}$ & Unpredictability \\
\hline
\end{tabular}


Tabelle 2: Zuordnung der Werte des Agilen Manifests zu den acht Octalysis Core Drives

\begin{tabular}{ll}
\hline Werte des Agilen Manifests (mit Diskretisierungen): & Zugeordnete Core Drives: \\
\hline $\begin{array}{l}\text { Individuen (individuelle Freiheit) und Interaktionen (Kommunikation) } \\
\text { mehr als Prozesse und Werkzeuge }\end{array}$ & $\begin{array}{l}\text { Empowerment und } \\
\text { Social Influence }\end{array}$ \\
\hline $\begin{array}{l}\text { Funktionierende Software (als Ziel für Module und Projekte) mehr als } \\
\text { umfassende Dokumentation }\end{array}$ & $\begin{array}{l}\text { Accomplishment und } \\
\text { Epic Meaning }\end{array}$ \\
\hline $\begin{array}{l}\text { Zusammenarbeit mit dem Kunden mehr als Vertragsverhandlung (Kom- } \\
\text { munikation zur Vermeidung von Misserfolgen) }\end{array}$ & $\begin{array}{l}\text { Social Influence und } \\
\text { Avoidance }\end{array}$ \\
\hline $\begin{array}{l}\text { Reagieren auf Veränderungen (kreativ sein in unerwarteten Situationen) } \\
\text { mehr als Befolgen eines Plans }\end{array}$ & $\begin{array}{l}\text { Empowerment und } \\
\text { Unpredictability }\end{array}$ \\
\hline
\end{tabular}

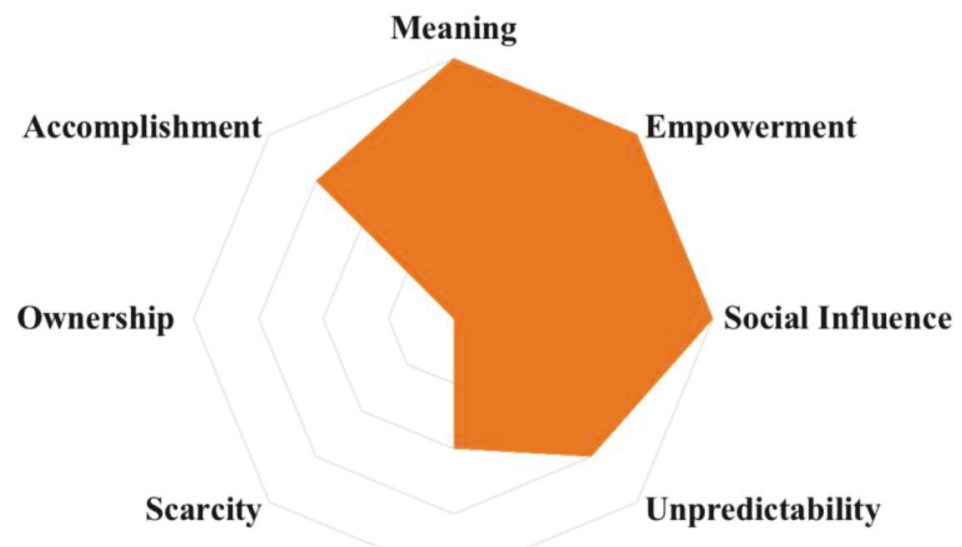

Avoidance

Abbildung 1: Hypothetisches Motivationsprofil des Agilen Manifests

Die Ergebnisse des Mappings sind in dem folgenden Octalysis-Diagramm in Abbildung 1 visualisiert. Das Mapping zeigt, dass es grundsätzlich sowohl beabsichtigte (Accomplishment, Meaning, Empowerment, Social Influence, Unpredictability, Avoidance), als auch nicht beabsichtigte (Ownership, Scarcity) Core Drives des Agilen Manifests für die Arbeit mit agilen Methoden gibt. 


\section{Quantitative Umfrage mit Scrum-Teammitgliedern}

Um zu analysieren, ob beabsichtigte sowie nicht beabsichtigte Core Drives existieren und um mögliche Unterschiede zum Agilen Manifest zu identifizieren, wird im folgenden Schritt eine Online-Befragung mit Scrum-Teammitgliedern durchgeführt.

In je einer Aussage zu den acht Core Drives wird die Zustimmung zum Vorhandensein der einzelnen Core Drives im Scrum-Prozess erhoben. Beispielsweise lautet die Aussage für den Core Drive Epic Meaning: "Im Scrum-Prozess hat der Mitarbeiter das Gefühl, einen höheren Sinn für seine Arbeit zu haben." Um ein gemeinsames Verständnis der Aussagen zu schaffen, werden zusätzlich Beispiele für die Umsetzung des jeweiligen Core Drives gegeben. Zum Beispiel sehen die Teilnehmer nach dem Epic MeaningStatement Umsetzungsmöglichkeiten wie "humanitäres Engagement, Gefühl der Einzigartigkeit oder elitäre Gruppenzugehörigkeit". Die Teilnehmer geben ihre Zustimmung zu diesen acht Aussagen auf einer Skala von null bis zehn an ( $0=$ stimme überhaupt nicht zu; 10= stimme voll zu).

Die Ergebnisse dieser Umfrage sind in der prozentualen durchschnittlichen Zustimmung in Abbildung 2 dargestellt:

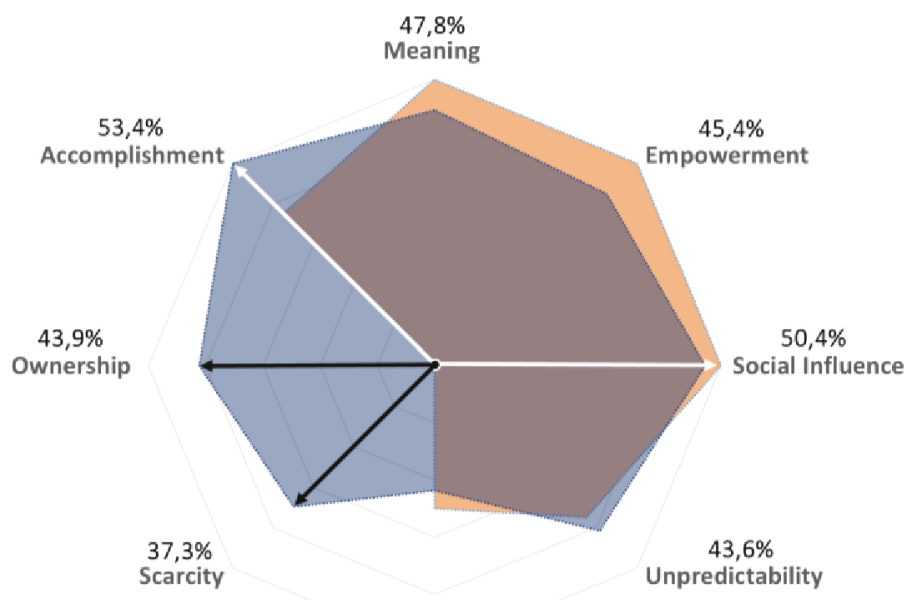

$23,3 \%$

Avoidance

Abbildung 2: Ergebnisse der qualitativen Befragung zur Erfüllung der Octalysis Core Drives bei der Arbeit im Scrum-Prozess $(N=71)$ 
Die Ergebnisse zeigen, dass der Scrum-Prozess im Allgemeinen die beabsichtigten Core Drives des Agilen Manifests unterstützt.

Neben den beabsichtigten sind auch die nicht beabsichtigten Core Drives Ownership und Scarcity in den Befragungsergebnissen vorhanden. Der Hauptunterschied zu den beabsichtigten Core Drives besteht darin, dass sich diese Motivationsfaktoren ohne die Werte und Prinzipien des Agilen Manifest entwickelt haben.

Grundsätzlich ergeben sich zwei verschiedene Entwicklungsmöglichkeiten für die Gamification-Strategien:

1. Verbesserung der Motivation von Scrum-Teammitgliedern durch Implementierung von Ownership und Scarcity Gamification-Techniken, um die bisher nicht vorhandenen Motivationsfaktoren strukturiert zu ergänzen.

2. Verbessern der Motivation von Scrum-Teammitgliedern durch die Implementierung von Accomplishment und Social Influence Gamification-Techniken, da dies die am stärksten vertretenen Motivationsfaktoren sind, um den bereits vorhandenen Einfluss zu nutzen.

Die neunte Frage der Umfrage beginnt mit einem kurzen Einführungstext. Die Teilnehmer werden gebeten, die Core Drives zu markieren, die sie in jeder einzelnen ScrumPhase am meisten motivieren. Hierfür wird eine 5x8-Matrix verwendet, die die Phasen Scrum Planning, Sprint Phase, Daily Scrum, Sprint Review und Sprint Retrospective im Vergleich zu den motivierenden Core Drives visualisiert.

Die Umfrage-Teilnehmenden haben die Möglichkeit, für jede Scrum-Phase mehrere Core Drives auszuwählen. Die Möglichkeit, mehrere Antworten geben zu können ist enthalten, da Menschen in der Regel durch mehrere Faktoren motiviert werden (Chou 2016).

Die Ergebnisse der Umfrage sind in Abbildung 3 dargestellt.

Insgesamt ist Social Influence der stärkste Core Drive, was zu den vorherigen Ergebnissen aus der Umfrage passt. Dies hängt mit den Eigenschaften agiler Methoden zusammen, da diese flache Hierarchien, Transparenz und Flexibilität anstreben. Folglich hat die Kommunikation in agilen Methoden im Allgemeinen und in Scrum im Besonderen eine höhere Priorität als in klassischen Entwicklungsmethoden. Insbesondere das Daily Scrum und die Sprint Retrospektive spielen eine wichtige Rolle in Bezug auf Social Influence. Zusätzlich ist Social Influence auch während des Sprint Reviews wichtig. Die Analyse der Ergebnisse für jede einzelne Scrum-Phase zeigt, dass der Core Drive Epic Meaning vor allem in den Off-Sprint-Events Sprint Planning, Sprint Review und Sprint 
Retrospective vorhanden ist. Accomplishment ist in der Sprint-Phase und den integrierten Daily Scrums stark vertreten. Empowerment und Ownership gibt es vor allem während des Sprint Plannings und des Sprints selbst. Scarcity wird im Allgemeinen als weniger wichtig wahrgenommen. Sie kann jedoch Teammitglieder während der OffSprint-Events motivieren.

Mit diesen Umfrageergebnissen können die Gamification-Strategien den passendsten Scrum-Phasen zugeordnet werden.

1. Steigerung der Motivation des Scrum-Teams durch Implementierung von Ownership-Gamification-Techniken in der Sprint-Phase und Scarcity-GamificationTechniken in den Off-Sprint-Events, um nicht-beabsichtigte Motivationsfaktoren zu strukturieren.

2. Steigerung der Motivation des Scrum-Teams durch Accomplishment-Gamification-Techniken in der Sprint-Phase sowie im Daily Scrum und Social InfluenceGamification-Techniken im Daily Scrum sowie in der Sprint-Retrospektive, um die stark vertretenen Motivationsfaktoren zu unterstützen.

\begin{tabular}{|c|c|c|c|c|c|}
\hline & $\begin{array}{c}\text { Sprint } \\
\text { Planning }\end{array}$ & Sprint & Daily Scrum & $\begin{array}{c}\text { Sprint } \\
\text { Review }\end{array}$ & $\begin{array}{c}\text { Retro- } \\
\text { spective }\end{array}$ \\
\hline Meaning & $40,43 \%$ & $25,58 \%$ & $30,43 \%$ & $52,17 \%$ & $63,64 \%$ \\
\hline Accomplishment & $34,04 \%$ & $65,12 \%$ & $63,04 \%$ & $45,65 \%$ & $38,64 \%$ \\
\hline Empowerment & $48,94 \%$ & $51,16 \%$ & $21,74 \%$ & $6,52 \%$ & $20,45 \%$ \\
\hline Ownership & $51,06 \%$ & $60,47 \%$ & $32,61 \%$ & $45,65 \%$ & $43,18 \%$ \\
\hline Social Influence & $48,94 \%$ & $39,53 \%$ & $69,57 \%$ & $54,35 \%$ & $65,91 \%$ \\
\hline Scarcity & $19,15 \%$ & $4,65 \%$ & $8,70 \%$ & $19,57 \%$ & $20,45 \%$ \\
\hline Unpredictability & $19,15 \%$ & $27,91 \%$ & $19,57 \%$ & $10,87 \%$ & $20,45 \%$ \\
\hline Avoidance & $12,77 \%$ & 9,30\% & $13,04 \%$ & $19,57 \%$ & $11,36 \%$ \\
\hline
\end{tabular}

Abbildung 3: Ergebnisse der Umfrage zu den wichtigsten Core Drives in jeder Scrum-Phase $(N=47)$

\section{Fazit}

Die vorliegende Forschung zeigt die methodische Entwicklung von Gamification-Strategien zur Motivationssteigerung von Scrum-Entwicklungsteams. Die Analyse des Agilen Manifests als Grundlage agiler Methoden, identifiziert beabsichtigte und nicht be- 
absichtigte Kernantriebe des Scrum-Prozesses. Eine Befragung von Scrum-Teammitgliedern führte zu einem Motivationsprofil und anschließend zu der Verteilung der Core Drives auf die Scrum-Phasen. Mit diesen Ergebnissen war die Zuordnung der Core Drives zu den Scrum-Phasen möglich, um Gamification-Strategiekonzepte zu bilden.

Zukünftig müssen konkrete Gamification Techniken zur Implementierung konzipiert werden, um die Anwendung in Scrum-Entwicklungsteams möglich zu machen. Eine besondere Bedeutung wird in diesem Zusammenhang auch der Effektivitätsmessung der Maßnahmen zugeschrieben. Dies ist ebenfalls Gegenstand aktueller Forschung.

\section{Literaturverzeichnis}

Anderie, L. (2017): Gamification, Digitalisierung und Industrie 4.0, In: Business and Economic, Springer Verlag, ISBN: 978-3658-19864-0

Beck, K.; Beedle, M.; van Bennekum, A.; Cockburn, A.; Cunningham, W.; Fowler, M.; Grenning, J.; Highsmith, J.; Hunt, A.; Jeffries, R.; Kern, J.; Marick, B.; Martin, R. C.; Mellor, S.; Schwaber, K.; Sutherland, J.; Thomas, D. (2001): Manifest für Agile Softwareentwicklung. Prinzipien hinter dem Agilen Manifest, Beitrag im Internet: http://agilemanifesto.org/iso/de/principles.html, abgerufen am 24.03.2021

Beedle, M.; Devos, M.; Sharon, Y.; Schwaber, K.; Sutherland, J. (1999): SCRUM: An extension pattern language for hyperproductive software development. In: Pattern Languages of program design (1999) Nr. 4, S. 636-651.

CollabNet Inc. (2019): 13th Annual State of Agile Report. CollabNet Inc. 2019. https://www.stateofagile.com/\#ufh-i521251909-13th-annual-state-of-agile-report/473508, zuletzt abgerufen am 29.10.2020

Chou, Y. (2016): Actionable Gamification. Octalysis Media, Milpitas, CA, USA

Deterding, S., et al. (2011): From Game Design Elements to Gamefulness: Defining "Gamification". New York, NY: ACM. Beitrag im Internet: http://dl.acm.org/citation.cfm?id=2181037, abgerufen am 14.05.2020

Dräther, R.; Koschek, H.; Sahling, C. (2019): Scrum - kurz \& gut. Heidelberg. O'Reilly, ISBN 3960102526

Ellenberger, T.; Harder, D.; Brechbühler Pešková, M. (2020): Gamification in Unternehmen. In: Schellinger, Jochen; Tokarski, Kim Oliver; Kissling-Näf, Ingrid. Digitale Transformation und Unternehmensführung: Trends und Perspektiven für die Praxis, p. 55-81. Wiesbaden: Springer Fachmedien

Feldhusen J., Löwer, M., Bungert. F. (2009): Agile Methods for Design to Customer. In: Proceedings of the 17th International Conference on Engineering Design (ICED09), Stanford University, California, USA, 2009

Fleisch, H., Mecking, C. \& Steinsdörfer, E. (2018): Gamification4Good: Gemeinwohl spielerisch stärken (1. Aufl.). Edition Stiftung\&Sponsoring: v.1. Erich Schmidt Verlag. Berlin. ISBN: 9783503177967

Krieg, A. (2015): Bierdeckelskizzen - Scrum ist leicht aber nicht einfach. In: Projektmanagement und Vorgehensmodelle. Lecture Notes in Informatics p. 107-117 
Marczewski, A. (2015): Even ninja monkeys like to play. Gamification, game thinking \& motivational design, Gamified UK, Unabhängige Veröffentlichung, ISBN 1514745666.

Maximini, D. (2018): Scrum - Einführung in der Unternehmenspraxis. Von starren Strukturen zu agilen Kulturen. Berlin. Springer Gabler, 2018. ISBN 9783662563250

Morschheuser, B., Hamari, J., Werder, K. \& Abe, J. (2017): How to Gamify? A Method For Designing Gamification. In Proceedings of the 50th Hawaii International Conference on System Sciences, Hawaii International Conference on System Sciences 2017, https://doi.org/10.24251/HICSS.2017.155

Paetzold, K.; Schmidt, T. S. (2016): Agilität als Alternative zu traditionellen Standards in der Entwicklung physischer Produkte: Chancen und Herausforderungen. In: 27. Dfx-Symposium 2016

Reiners, T.; Wood, L. C. (2015): Gamification in Education and Business. Cham. Springer International Publishing, ISBN 9783-319-10207-8.

Zichermann, G. \& Cunningham, C. (2011): Gamification by design: Implementing game mechanics in web and mobile apps (1st ed.), O'Reilly Media. Sebastopol, CA, USA, ISBN: 978-1-449-39767-8

\section{Kontakt}

David Kessing, M. Sc. RWTH

Univ.-Prof. Dr.-Ing. Manuel Löwer

Fachgebiet Produktsicherheit und Qualität

Bergische Universität Wuppertal

Gaußstraße 20

42119 Wuppertal

www.psq.uni-wuppertal.de 